\title{
Antioxidants and Cancer: Theories, Techniques, and Trials in Preventing Cancer
}

\author{
Gloria M. Calaf $\mathbb{D},{ }^{1,2}$ Francisco Aguayo, ${ }^{3}$ Consolato M. Sergi $\mathbb{D}^{\mathrm{D}},{ }^{4}$ Angeles Juarranz $\mathbb{D},{ }^{5,6}$ \\ and Debasish Roy ${ }^{7}$ \\ ${ }^{1}$ Instituto de Alta Investigación, Universidad de Tarapacá, Arica, Chile \\ ${ }^{2}$ Center for Radiological Research, Columbia University Medical Center, New York City, NY, USA \\ ${ }^{3}$ Departamento de Oncología Básico Clínica, Facultad de Medicina, Universidad de Chile, Santiago, Chile \\ ${ }^{4}$ Department of Laboratory Medicine and Pathology, University of Alberta, Edmonton, AB, Canada \\ ${ }^{5}$ Laboratory of Photocarcinogenesis, Department of Biology, Faculty of Sciences, Autonomous University of Madrid, Madrid, Spain \\ ${ }^{6}$ Instituto Ramón y Cajal de Investigación Sanitaria (IRYCIS), Madrid, Spain \\ ${ }^{7}$ Laboratory of Molecular Carcinogenesis, Department of Natural Science, Shirley J. Hinds Allied Health Science Complex, Hostos \\ Community College of the City University of New York, Bronx, NY, USA
}

Correspondence should be addressed to Gloria M. Calaf; gmc24@columbia.edu

Received 20 March 2018; Accepted 20 March 2018; Published 20 May 2018

Copyright ( 2018 Gloria M. Calaf et al. This is an open access article distributed under the Creative Commons Attribution License, which permits unrestricted use, distribution, and reproduction in any medium, provided the original work is properly cited.

Compounds of natural origin and their derivatives play an increasingly important role in medicine and pharmacology. Approximately $60 \%$ of therapeutic drugs used in the treatment of cancer are compositions comprising natural compounds and/or their derivatives.

C. G. Vazhappilly and H. P. Vasantha Rupasinghe demonstrated that an apple flavonoid fraction (AF4) can protect oxidative DNA damage in vitro and facilitate repair mechanisms in normal human bronchial epithelial cells exposed to carcinogen-induced DNA damage as nicotinederived nitrosamine ketones, nitrosamine ketones-acetate, methotrexate, and cisplatin. When DNA damage and repair mechanisms were evaluated, it was found that AF4 pretreated cells showed lower cytotoxicity, total ROS generation, and DNA fragmentation along with consequent inhibition of DNA tail moment after phosphorylation of histone $(\gamma$-H2AX).

A. Ortiz-Espín et al. evaluated an extract of an Antarctic plant Deschampsia antarctica (EDA) in young human fibroblasts exposed to $\mathrm{H}_{2} \mathrm{O}_{2}$ to survive in extreme conditions. They measured cell proliferation, viability, and senescenceassociated $\beta$-galactosidase (SA- $\beta-\mathrm{Gal})$. They found that EDA per se promoted cell proliferation and viability and increased the expression of antisenescence-related markers. They also tested the expression of several senescenceassociated proteins including redox protein thioredoxin, sirtuin 1, and lamin $\mathrm{A} / \mathrm{C}$ and the replicative protein PCNA. Then, they induced senescence in human fibroblasts and they found that an EDA treatment significantly inhibited the increase in SA- $\beta$-Gal levels induced by $\mathrm{H}_{2} \mathrm{O}_{2}$ and promoted the expression of sirtuin 1 and lamin $\mathrm{A} / \mathrm{C}$ proteins. The results suggest that EDA protects human fibroblasts from cellular senescence, pointing to this compound as a potential therapeutic agent to treat or prevent skin senescence.

G. Carrasco-Torres et al. used quercetin, a flavonoid considered as chemopreventive agent in different types of cancer. They demonstrated that quercetin was able to prevent and reverse rat liver preneoplastic lesions when using the modified resistant hepatocyte model by downregulating the expression of EGFR and phosphorylating the status of Src-1, STAT5, and Sp-1. Then, they concluded that quercetin reversed preneoplastic lesions and had a chemopreventive effect on the liver of rats. Plant-based compounds are still researched for their anticancer activity and for their quantity in plants. Therefore, the modern chromatographic methods are applied to quantify them 
in plants, as the ultraperformance liquid chromatographytandem mass spectrometry.

T. Kubrak et al. studied the effect of 20 coumarin derivatives on the cytotoxicity and expression of encoding proteins responsible for multidrug resistance (MDR) and genes involved in such resistance in cancer cells. Such genes are considered as the major cause of failure of cancer chemotherapy, demonstrated as overexpression of membrane transporters primarily from the $\mathrm{ABC}$ family which actively remove cytostatics from the tumor cell. They studied proteins as MDR1, MRP, and $L R P$ and genes as BCRP in the presence and absence of mitoxantrone in 5 cell lines derived from the human hematopoietic system and found that leukemia cells exhibited a multidrug resistance phenotype.

M. Mehdi et al. evaluated glutamate and glucose metabolism through GDH and LDH enzyme activity, oxidant, and antioxidative status among breast cancer patients from Addis Ababa, Ethiopia. Catalytic activities of glutamate dehydrogenase, lactate dehydrogenase, and oxidative stress index were significantly increased both in serum and cancerous tissues of breast cancer patients as compared to control groups of breast cancer patients. They concluded that catalytic activities of GDH and LDH among breast cancer patients were significantly higher than control groups and noncancerous tissues of breast cancer patient. A problem of cancer chemotherapy is the high cytotoxicity toward normal rapidly proliferating cells, especially the bone marrow. In order to mitigate side effects, modified therapeutic regimens such as combination therapy have been introduced.

A. Och et al. studied the content of alkaloids as sanguinarine, berberine, protopine, and chelidonine, unidentified in plant species known for their anticancer activity. Plantbased compounds are still researched for their anticancer activity and for their quantity in plants. Therefore, the modern chromatographic methods are applied to quantify them in plants, for example, UPLC-MS/MS (ultraperformance liquid chromatography-tandem mass spectrometry).

Gloria M. Calaf

Francisco Aguayo

Consolato M. Sergi

Angeles Juarranz

Debasish Roy 


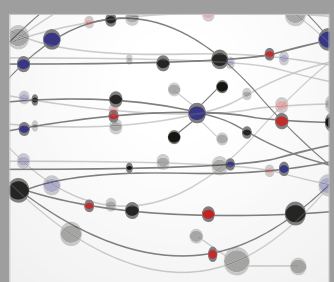

The Scientific World Journal
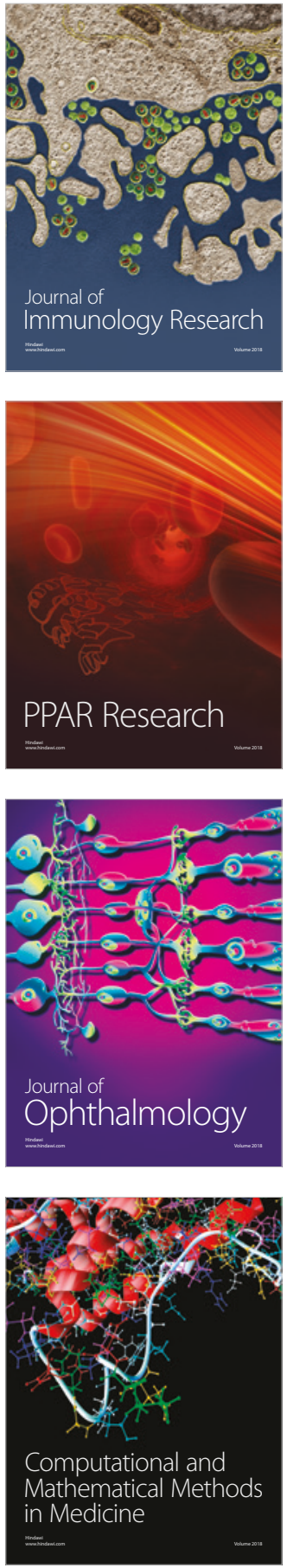

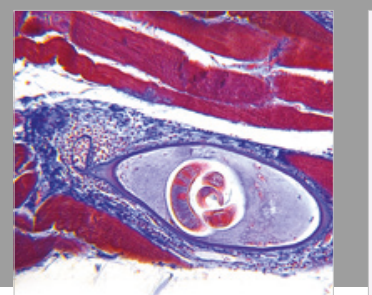

Gastroenterology Research and Practice

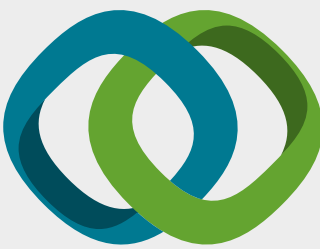

\section{Hindawi}

Submit your manuscripts at

www.hindawi.com
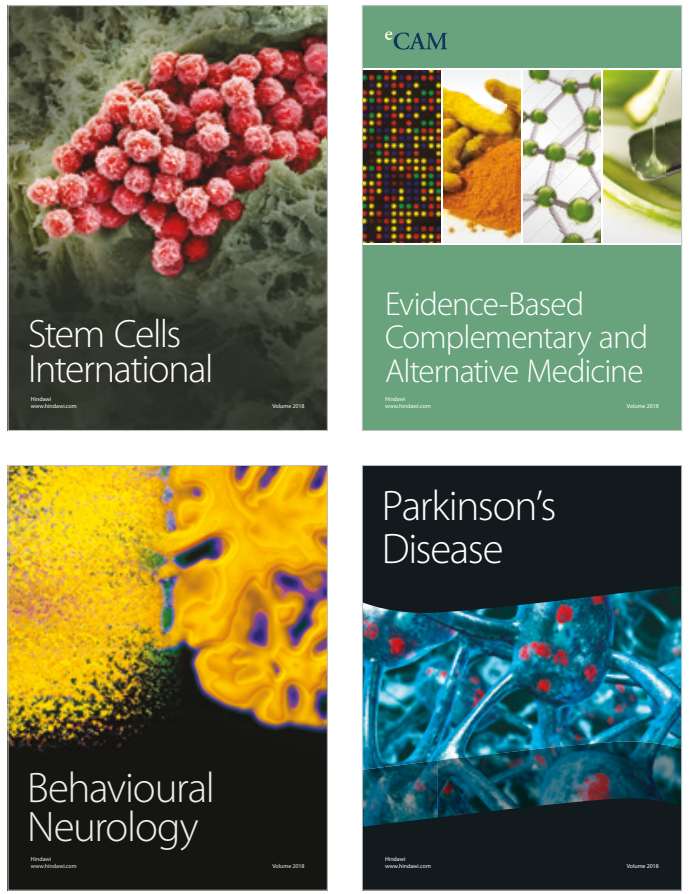

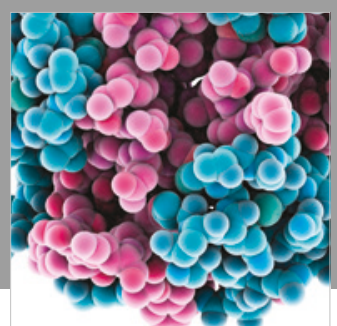

ournal of

Diabetes Research

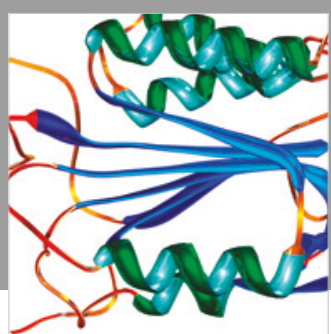

Disease Markers
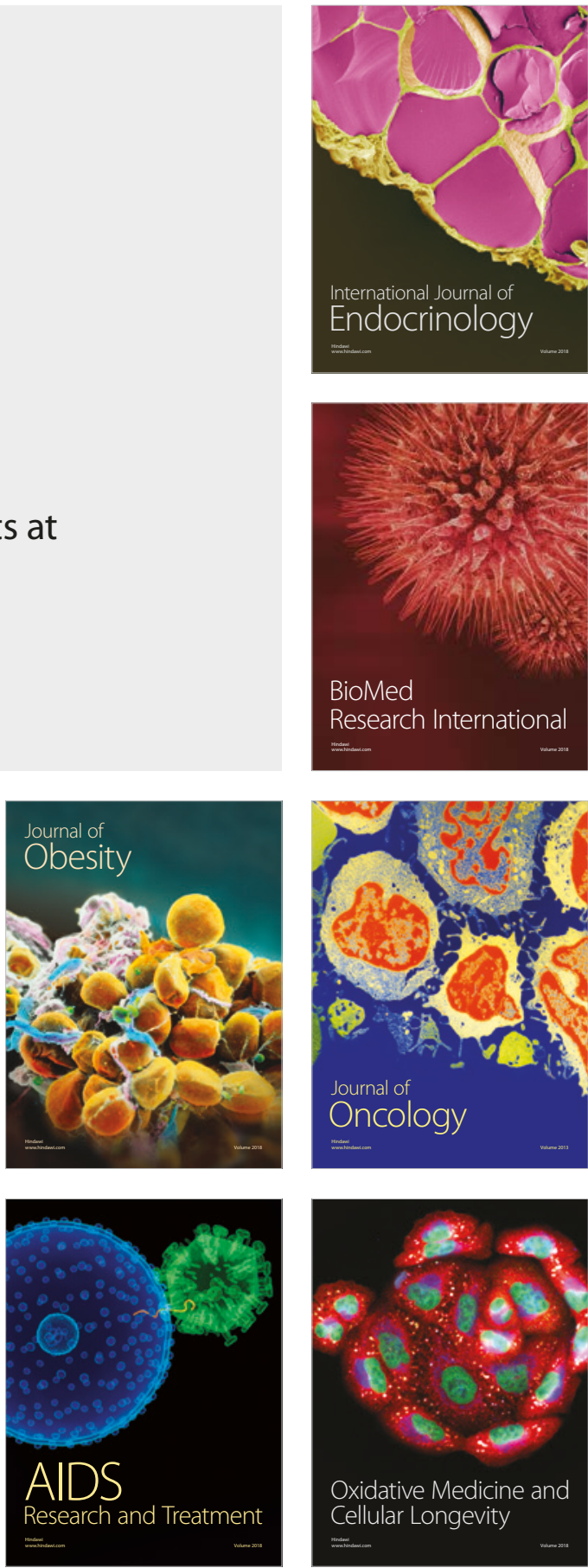